\title{
Nonstationary gas-dynamics and local heat transfer in the output channel of the turbocharger compressor
}

\author{
Leonid Plotnikov ${ }^{1, *}$, Nikita Grigor'ev ${ }^{1}$, and Nikolaj Kochev ${ }^{1}$ \\ ${ }^{1}$ Ural Federal University named after the first President of Russia B.N. Yeltsin, Turbines and engines \\ Department, 620002 Ekaterinburg, Mira str., 19, Russian Federation
}

\begin{abstract}
Thermomechanical characteristics of the gas flow at the turbocharger compressor outlet largely determine the quality of the intake process in piston engines with boost. The article presents the results of an experimental study of gas-dynamics and heat transfer of gas flows after compression in a turbocharger centrifugal compressor. A brief description of the experimental setup, the configuration of pipes under investigation, the measuring system and the experimental features are given. The studies were carried out on a free compressor, i.e. without considering the piston part. Different conditions in the compressor outlet channel were created by installing special nozzles with different hydraulic resistances. It has been established that the local heat transfer increases from 23 to $46 \%$ with an increase in the turbocharger rotor speed, depending on the outlet channel configuration. It should be noted that an increase in rotor speed is also accompanied by an increase in air flow through the channel. The increase in flow rate was from 10 to $42 \%$.
\end{abstract}

\section{Introduction}

Most modern internal combustion engines (ICE) are equipped with turbochargers (TC), i.e. a supercharging system based on a gas connection between the piston part and the scapular machine. Therefore, the mutual agreement of the thermomechanical characteristics of gas flows between the ICE and TC pipelines is an actual topic in the development of engine building. Today, the receipt of new data on the features of gas-dynamics and heat transfer in gas-air tracts of turbocharged engines is an important task, since the efficiency of power machines based on ICEs depends on the perfection of these processes [1-3]. Modern research in this field is devoted to the study of the parameters of gas flows at the inlet and outlet of the TC turbine [4-6], heat transfer inside the compressor [7], the thermal effect of the turbine on the compressor in the composition of the TC [8] and ways to improve the efficiency of the turbocharger $[9,10]$.

This article presents the results of an experimental study of gas-dynamics and heat transfer of gas flows in the output channel after compression in a centrifugal compressor of a turbocharger.

\footnotetext{
* Corresponding author: leonplot@mail.ru
} 


\section{Experimental setup and measuring equipment description}

The experimental setup was designed and manufactured to study the gas-dynamics and heat exchange of gas flows in the output channel behind the turbocharger compressor without taking into account the influence of the piston part (ICE) (Fig.1). The setup contains a test turbocharger (this is a Russian TK with the designation TKR6), which consists of a centrifugal compressor and a single-stage turbine on one shaft. The TC rotor is rotated by compressed air entering the turbine blades. The source of compressed air is an external piston compressor. It should be noted that the air temperature for driving the TC rotor is approximately equal to the air temperature in the compressor under test. The turbocharger rotor speed of the is regulated by changing the flow rate and air pressure in front of the turbine and ranges from 30000 to $60000 \mathrm{rpm}$.

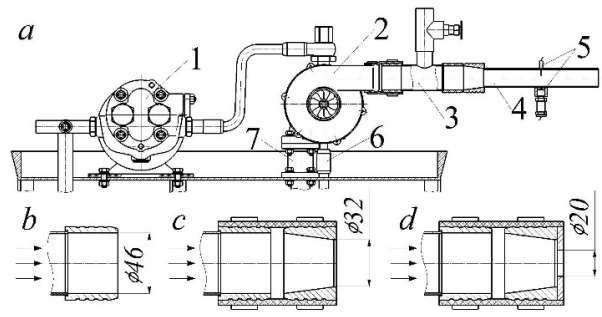

Fig. 1. The scheme of the experimental setup and output nozzles: 1 - oil pump; 2 - turbocharger; 3 outlet nozzle; 4 - investigated channel; 5 - control section with the sensors; 6 - oil drain pipe; 7 pipeline for supplying compressed air to drive the turbine; $a$ - experimental setup; $b$ - outlet nozzle with diameter of $46 \mathrm{~mm} ; c$ - outlet nozzle with confuser and diameter of $32 \mathrm{~mm}$; $d$ - outlet nozzle with a diaphragm and diameter of $20 \mathrm{~mm}$

The intake system of a turbocharged engine can be viewed as two elements: a turbocharger and a hydraulic network consisting of an intake pipe and a piston engine. Thus, the gas-dynamic characteristics of the network can be changed by longitudinal profiling of the compressor output channel section. In this study, nozzles with different hydraulic resistances were used to simulate a network (ICE), the geometric dimensions of which are shown in Fig. $1 b$ - $d$.

The automated system for collecting and processing experimental data was created on the basis of an analog-to-digital converter for conducting research. The constanttemperature thermal anemometer was used to determine the local air flow rate $\left(w_{x}\right)$ and the heat transfer coefficient $\left(\alpha_{x}\right)$. Nichrome filament was the sensitive element of the thermal anemometer sensors in both cases. The time constant of both thermal anemometers was about $2 \mathrm{~ms}$. The systematic error of the velocity $\left(w_{x}\right)$ measurements was $5.4 \%$, and the local coefficient of heat transfer was $10 \%$. The method of measuring the instantaneous values of the local velocity and the heat transfer coefficient is described in [11]. WIKA pressure sensor was used to measure instantaneous values of static pressure $p_{x}$ in the flow after the compressor; the sensor speed was less than $1 \mathrm{~ms}$. The pressure measurement error was $\pm 0,25 \%$. The digital non-contact tachometer was used to measure the turbocharger rotor speed. The control section with the sensors was located at a distance of $300 \mathrm{~mm}$ from the flange of the turbocharger compressor outlet channel.

\section{Thermomechanics in the output channel of the turbocharger compressor}

The dependences of local pressure $p_{x}$, velocity $w_{x}$ and local heat transfer coefficient $\alpha_{x}$ on the time in the compressor output channel with a confuser (diameter $32 \mathrm{~mm}$ ) are shown in 
Fig. 2 as an example. Similar data were obtained for all configurations of the compressor outlet channel in the TC rotor speed range from 30000 to $60000 \mathrm{rpm}$.

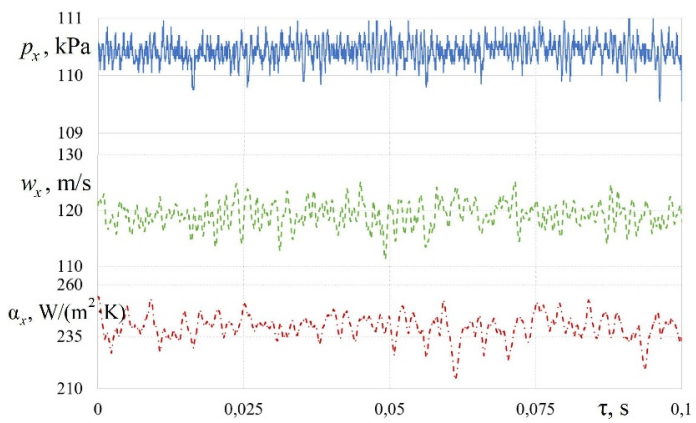

Fig. 2. The dependences of the pressure $p_{x}$, the flow velocity $w_{x}$ and the local heat transfer coefficient $\alpha_{x}$ on the time $\tau$ in the output channel (confuser with $32 \mathrm{~mm}$ ) of the centrifugal compressor for the TC rotor speed equal to $42000 \mathrm{rpm}$

It has been established that the air flow velocity has maximum values at the level of $130-140 \mathrm{~m} / \mathrm{s}$ with free outlet from the compressor (channel with a diameter of $46 \mathrm{~mm}$ ). In this case, the pressure in the channel has approximately the same value of about $100 \mathrm{kPa}$ at all rotational speeds $n_{\text {Tc }}$. This is explained by the insignificant value of the channel resistance with free outlet of an air. The maximum values of the static pressure of the air flow increase to $110 \mathrm{kPa}$ in the case of an outlet channel with a confuser (diameter $32 \mathrm{~mm}$ ). At the same time, air flow velocity values are reduced to $105-120 \mathrm{~m} / \mathrm{s}$ with an increase in the turbocharger rotor speed compared to free outlet. The static pressure of the air flow increases to $130 \mathrm{kPa}$, and the flow velocity decreases substantially and ranges from 55-85 $\mathrm{m} / \mathrm{s}$ when the diaphragm (diameter $20 \mathrm{~mm}$ ) is installed in the compressor outlet channel. The trend is maintained: instantaneous values of a pressure and flow velocity in the compressor output channel increase with the TC rotor speed.

The analysis of the compressor consumption characteristics with different output channel configurations was carried out. It is established that the air flow is reduced by an average of $15 \%$ when installing a confuser $(32 \mathrm{~mm})$ in the compressor outlet channel, compared to a free outlet $(46 \mathrm{~mm})$; and the average air flow rate is decreased up to $50 \%$ when setting the diaphragm $(20 \mathrm{~mm})$ in the output channel. At the same time, the increase in air flow through the compressor output channel is observed with the increase in the TC rotor speed, which is typical for all the studied configurations. Thus, an increase in air flow is about $10 \%$ with a free outlet, with an outlet channel with a confuser $-27 \%$, with a channel with a diaphragm $-40 \%$.

The change in gas-dynamic conditions in the compressor output channel affects the heat exchange intensity (Fig. 3). It has been established that the value of the heat transfer coefficient increases with the growth of the TC rotor speed, which is typical for all configurations of the compressor output channel. It should be noted that the increase in the $\alpha_{x}$ exceeds the increase in air consumption. For example, the increase in air flow through the output channel was approximately $10 \%$ with a free outlet from the compressor, while the heat transfer coefficient increased by $23.4 \%$. A similar trend is typical for other channel configurations. As expected, the decrease in the heat transfer intensity occurs on average by $26 \%$ with the installation of a confuser $(32 \mathrm{~mm})$ in the compressor outlet channel as compared to the free outlet, and the heat transfer intensity decreases to $70 \%$ when the diaphragm $(20 \mathrm{~mm})$ is installed in the channel.

The above-considered thermal and mechanical characteristics of the air flow in the compressor outlet channel have a significant effect on the processes in the intake system of the supercharged engines [12]. 


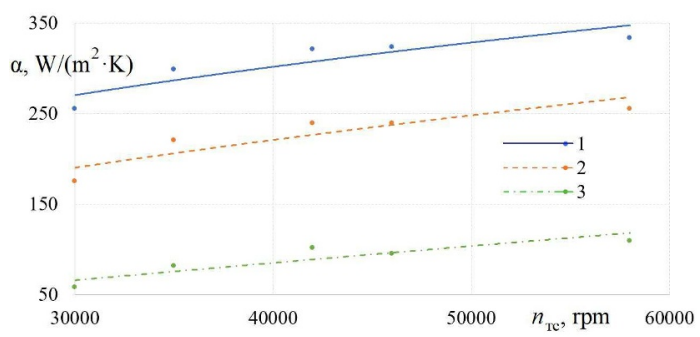

Fig. 3. Dependence of the local heat transfer coefficient $\alpha_{x}$ on turbocharger rotor speed $n_{\mathrm{Tc}}$ in the outlet channel of a free compressor for nozzles different configurations: 1 - free outlet $(46 \mathrm{~mm}) ; 2$ confuser (32 mm); 3 - diaphragm $(20 \mathrm{~mm})$

\section{Summary}

The following main conclusions can be drawn from the research:

- experimental setup for the investigation of gas-dynamics and heat transfer in the output channel of a turbocharger compressor was developed; influence of different configurations of the compressor output channel on the thermomechanical characteristics of gas flows is investigated;

- it has been established that an increase in air flow rate can be from 10 to $42 \%$ with an increase in the TC rotor speed under different configurations of the compressor channels;

- it has been shown that an increase in local heat transfer can be from 23 to $46 \%$ with increasing turbocharger rotor speed under different configurations of the compressor outlet channel;

- the obtained data can be used to increase the efficiency of thermal and mechanical processes in the intake systems of turbocharged engines.

The work has been supported by the Russian Science Foundation (grant No. 18-79-10003).

\section{References}

1. A. Romagnoli, A. Manivannan, S. Rajoo, M.S. Chiong, A. Feneley, A. Pesiridis, R.F. Martinez-Botas, Renewable Sustainable En. Rev. 79, 1442-1460 (2017).

2. R.D. Burke, C.D. Copeland, T. Duda, M.A. Rayes-Belmote, J. of Eng. Gas Turb. Power. 138(9), 092602 (2016).

3. S. Zhu, S. Liu, S. Qu, K. Deng, Energy. 140, 488-505 (2017).

4. L. Huang, C. Ma, Y. Li, J. Gao, M. Qi, Appl. Therm. Eng. 141, 1080-1091 (2018).

5. B. Franzke, P. Adomeit, T. Uhlmann, J. Scharf, S. Pischinger, Int. J. Engine Res. 19(4), 449-460 (2018).

6. L.V. Plotnikov, B.P. Zhilkin, Y.M. Brodov, IOPConf.Ser.: J. Phys. 891, 012154 (2017)

7. G. Tanda, S. Marelli, G. Marmorato, M. Capobianco, Appl. Energy. 193, 531-539 (2017).

8. M. Schinnerl, J. Ehrhard, M. Bogner, J. Seume, J. of Eng. Gas Turb. Power. 140(2), 022301 (2018).

9. C. Avola, C.D. Copeland, R.D. Burke, C.J. Brace, Energy. 134, 743-756 (2017).

10. M. Buchman, D. Ramanujan, A.G. Winter, SAE Int. J. of Engines. 11(4) (2018).

11. L.V. Plotnikov, B.P. Zhilkin, Int. J. Heat Mass Transfer. 115, 1182-1191 (2017).

12. L.V. Plotnikov, IOP Conf. Ser.: J. Phys. 899, 042008 (2017). 